\title{
THE DISTRIBUTION OF Li-LIKE IONS IN THE LOCAL BUBBLE
}

\author{
Miguel A. De Avillez ${ }^{1,2}$ ANd Dieter Breitschwerdt ${ }^{2}$ \\ ${ }^{1}$ Department of Mathematics, University of Évora, R. Romão Ramalho 59, 7000 Évora, Portugal; mavillez@ galaxy.lca.uevora.pt \\ 2 Institut für Astronomie, University of Vienna, Türkenschanzstraße 17, A-1180 Vienna, Austria; breitschwerdt@astro.univie.ac.at \\ Received 2008 January 3; accepted 2009 April 8; published 2009 May 14
}

\begin{abstract}
We study, by means of three-dimensional adaptive mesh refinement simulations, the spatial distribution of the Li-like ions C IV, Nv, and O vi inside the Local Bubble (LB), powered by 19 missing stars of subgroup B1 of the Pleiades suggested as responsible for its origin. The ions $\mathrm{C}$ IV, N v, and O VI, and the distribution of their column density ratios $(N(\mathrm{C} \mathrm{IV}) / N(\mathrm{O} \mathrm{VI})$ and $N(\mathrm{Nv}) / N(\mathrm{O} \mathrm{VI}))$ in the simulations are monitored through lines of sight (with lengths as large as $150 \mathrm{pc}$ ) emanating from the Solar position. The main results are: (i) there is a weak distribution of $\mathrm{CIV}_{\mathrm{IV}}$ and $\mathrm{NV}$ in the regions where $\mathrm{OVI}$ is strong, (ii) there is a small amount of $\mathrm{C}_{\mathrm{IV}}$ and $\mathrm{NV}$ ions inside the bubble translating into upper limits of -1.0 of the ratios $\log [N(\mathrm{C}$ IV $) / N(\mathrm{O}$ VI $)]$ and $\log [N(\mathrm{~N}$ v $) / N(\mathrm{O}$ VI $)]$, consistent with observations, and (iii) with the lack of heat conduction, turbulent mixing, due to the strong shear between the hotter and cooler regions inside the LB, is responsible for the generation and distribution of these ions.
\end{abstract}

Key words: hydrodynamics - ISM: bubbles - ISM: individual (Local Bubble) - ISM: structure - supernova remnants - turbulence

\section{INTRODUCTION}

There has been an ongoing discussion about the plasma conditions in the Local Bubble (LB), mainly because fitting $\mathrm{X}$-ray and extreme ultraviolet (EUV) observations with models based on collisional ionization equilibrium (CIE) resulted in severe mismatches, notably in a large number of soft X-ray (SXR) lines that were absent in the observations (see, e.g., McCammon et al. 2002; Hurwitz et al. 2005). The model of Breitschwerdt \& Schmutzler (1994) tried to explain this deficit by a somewhat cooler, "overionized" plasma due to delayed recombination of a fast adiabatically expanding bubble. The predictions of such a model based on non-equilibrium ionization (NEI), however, crucially depend on the thermodynamic path of the plasma, i.e., on the detailed underlying astrophysical model. Although X-ray observations are important for determining the LB plasma state, it is difficult to spatially and spectrally disentangle very local (due to solar wind charge exchange reactions, e.g., Lallement 2004) LB, and more distant Galactic contributions. On the other hand, UV absorption line data have much more detailed spatial information and can be very useful for constraining the ionization state and temperature distribution of the LB plasma.

High-resolution CIE simulations of the LB powered by the missing stars of the subgroup B1 of Pleiades (Berghöfer \& Breitschwerdt 2002; Fuchs et al. 2006) within a turbulent interstellar medium (ISM; Breitschwerdt \& de Avillez 2006, hereafter BA06) reproduced the clumpy distribution of O VI, its column density and dispersion inside the LB cavity as observed by FUSE (Oegerle et al. 2005; Savage \& Lehner 2006) and COPERNICUS (Jenkins 1978; Shelton \& Cox 1994). The match of the simulated $N(\mathrm{OVI})$ with the observed values indicates that the last supernova ( $\mathrm{SN}$ ) occurred $0.5 \mathrm{Myr}$ ago; that is, the LB has an age of $\sim 14.5$ Myr. It needs to be emphasized that the recombination timescales are different for different ions and their ionization stages, respectively, so that global CIE conditions cannot be guaranteed. The only way to test CIE validity is to perform a full-fledged NEI model, which is presently in progress. A prior step is to check the CIE model's consistency with column densities and their ratios of other
Li-like ions, e.g., C IV and Nv. Moreover, our simulations can help us to unravel the ionization mechanisms responsible for their origin. Observations along lines of sight (LOS) with lengths $<150 \mathrm{pc}$, that is, sampling gas in the LB, showed (i) a lack of $\mathrm{C} I V$ and $\mathrm{NV}$ with upper $3 \sigma$ limits on their column densities of $<7 \times 10^{11} \mathrm{~cm}^{-2}$ and $3 \times 10^{12} \mathrm{~cm}^{-2}$, respectively, and (ii) $\log [N(\mathrm{C}$ IV $) / N(\mathrm{O}$ vI $)]<-0.9 \pm-02$ and $\log [N(\mathrm{~N} \mathrm{v}) /$ $N(\mathrm{O}$ VI) $]<-1.0$ (see, e.g., York 1977; Bruhweiler et al. 1980; Bertin et al. 1995; Huang et al. 1995).

We extend our previous work to (i) perform a detailed analysis of these ions in the simulated LB at present time, (ii) compare these results with observations from COPERNICUs, IUE, GHRS, and STIS instruments on board Hubble Space Telescope (HST), and (iii) discuss the possible mechanisms for their generation. This Letter is organized as follows: Section 2 summarizes the model setup and simulation. Section 3 deals with the Li-like ions distribution and their column density ratios inside the LB at present time. Section 4 closes the Letter with a discussion and conclusions.

\section{MODEL AND SIMULATION}

We model the evolution of the interacting LB and the neighboring Loop I superbubble (Egger \& Aschenbach 1995) in a patch of the Galaxy with $0 \leqslant(x, y) \leqslant 1 \mathrm{kpc}$ size in the Galactic plane, and $|z| \leqslant 10 \mathrm{kpc}$ perpendicular to it. Periodic boundary conditions are applied along the four vertical boundary faces, while outflow boundary conditions are imposed at the $z= \pm 10 \mathrm{kpc}$ faces. A finest adaptive mesh refinement resolution of $1.25 \mathrm{pc}$ and $2.5 \mathrm{pc}$ is used in the regions $|z| \leqslant 500 \mathrm{pc}$ and $|z|>500 \mathrm{pc}$, respectively.

As an initial condition for the ambient medium, we use density, energy, and velocity field data cubes from a previous run taken after $200 \mathrm{Myr}$ evolution time of the general ISM-after the disk-halo circulation and a global dynamical equilibrium have been established. We arbitrarily chose a site with sufficient mass to convert into the $\sim 81$ stars that formed the Sco Cen cluster (Egger 1998), with 239 stars having generated the Loop I superbubble, while the other $\sim 42$ will explode within the next $\sim 13$ Myr. The LB was carved out by $\sim 19 \mathrm{SNe}$ 


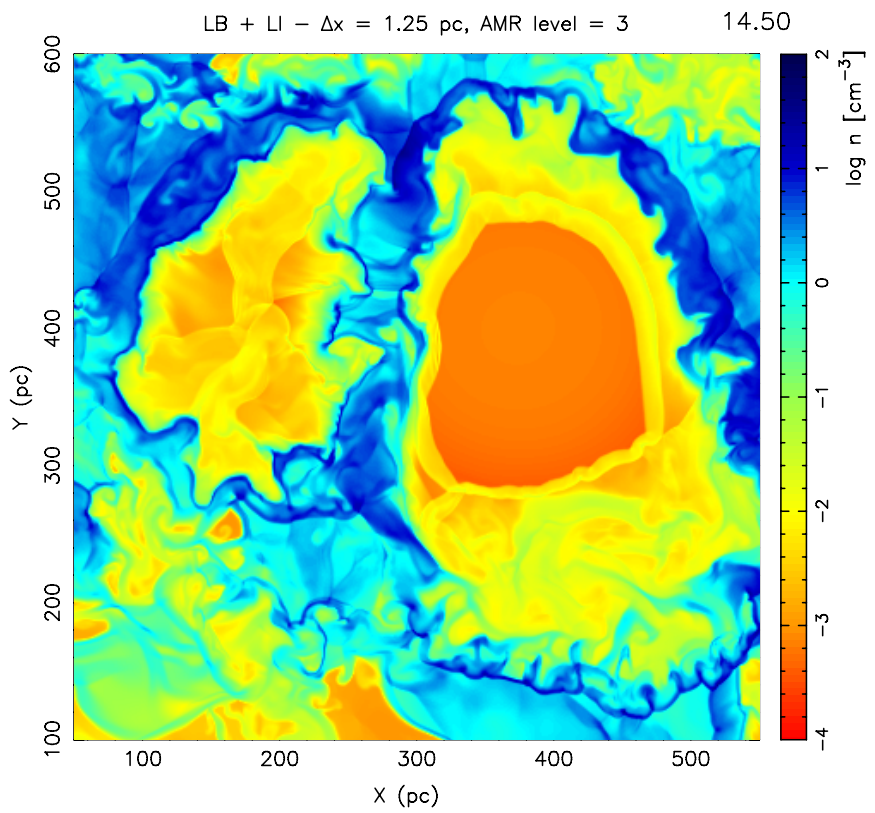

Figure 1. Zoom-in on the density distribution in the LB (left cavity) and Loop I (right cavity) superbubbles at $14.5 \mathrm{Myr}$ after the first SN occurrence in the solar vicinity.

(with masses between $\sim 19 M_{\odot}$ and $\sim 12 M_{\odot}$ ) belonging to the subgroup B1 of Pleiades, on its motion toward Sco Cen (Berghöfer \& Breitschwerdt 2002). Thus, we followed the trajectory of the B1 cluster, inferred from proper motions and distances according to Hipparcos data and supplemented by radial velocities, backwards in time, and set the explosions of "missing B1 members" at the end of their main-sequence life times (as estimated by Stothers 1972). The simulation time of this run was $30 \mathrm{Myr}$, which exceeds the stellar cluster ages.

The model further includes: (i) SNe types Ia and II occurring at the Galactic $\mathrm{SN}$ rate, (ii) self-consistent generation of stars in the disk using an efficiency of 5\% and the initial mass function for Galactic OB associations, (iii) a gravitational field of the stellar disk, (iv) radiative cooling assuming CIE and solar abundances (Anders \& Grevesse 1989; AG89), and (v) uniform background heating due to starlight varying with $z$. In de Avillez \& Breitschwerdt (2005), we determined that heat conduction plays a lesser role than turbulent mixing in the ISM, in general, and bubbles, in particular, and therefore have excluded heat conduction in the present simulations. For further details on the model setup and simulations, see BA06.

\section{RESULTS}

The successive SNe in the Solar neighborhood and in Sco Cen produce coherent bubble structures, which at first are spherical, but soon evolve, and grow, to deformed three-dimensional structures depending on the density and pressure distributions of the surrounding medium into which they expand. However, the LB cavity becomes progressively elongated, not only due to ambient medium conditions, but also due to the motion of the exploding stars along their path toward Sco Cen, reaching a size of 180 by 220 pc, while Loop I has a more spherical form (Figure 1). The figure displays the density distribution in and around the LB (left cavity) and Loop I (right cavity) superbubbles at time 14.5 Myr. Dark blue represents the high density and cooler material, while red represents low density (less than $\left.10^{-2} \mathrm{~cm}^{-3}\right)$ hot gas $\left(T>10^{6} \mathrm{~K}\right)$.
We study the spatial distribution of the Li-like ions CIV, Nv, and O VI both by means of density maps (Figure 2) that show a weak distribution of $\mathrm{CIV}$ and $\mathrm{NV}$ in the regions where $\mathrm{OVI}$ is strong, and LOS measurements of the ions' column densities. The LOS taken from the Sun's vantage point, located at $(175,400) \mathrm{pc}$ (center of the left bubble in Figure 1), cover the region $|z| \leqslant 10 \mathrm{pc}$, and span a range of $360^{\circ}$ when projected onto the Galactic midplane. The maximum LOS length is $150 \mathrm{pc}$ and the step length is $10 \mathrm{pc}$. The periodicity of the boundary conditions along the $x$ - and $y$-directions ensures the continuity with surrounding regions that are not calculated, thereby not affecting the column densities near the boundaries.

Figure 3 shows the angular distribution of the column density ratios of $\log [N(\mathrm{C} \mathrm{IV}) / N(\mathrm{O} \mathrm{vI})]$ (varying between -2.5 and 0.6 ; left panel) and $\log [N(\mathrm{~N} \mathrm{v}) / N(\mathrm{O} \mathrm{vI})]$ (varying between -1.7 and -0.5 ; right panel) for LOS (with lengths of 10, 30, 50, 70, 90 , and $110 \mathrm{pc}$ ) measurements. It is observed that the largest dispersion in $\log [N(\mathrm{C}$ IV $) / N(\mathrm{O}$ VI $)]$, up to 3 orders of magnitude, varies from location to location inside the cavity. Similar behavior is seen in the $\log [N(\mathrm{Nv}) / N(\mathrm{O} \mathrm{VI})]$ ratios, but with a smaller dispersion covering at most 1.5 orders of magnitude. These dispersion variations are a result of the dynamical processes, e.g., turbulent mixing, occurring in the cavity (see below), and, because of the assumption of CIE, are an indication of large temperature inhomogeneities. Twenty percent of the LOS have $\log [N(\mathrm{C} \mathrm{IV}) / N(\mathrm{O} \mathrm{VI})] \in[-0.5,1.0]$, while $80 \%$ and $64 \%$ of all LOS have values of $N(C$ IV $) / N(O$ VI $)<-0.5$ and $N(C$ IV $) / N(O \mathrm{VI})<-1.0$, respectively (Figure 4 , black histogram). For $N(\mathrm{Nv}) / N(\mathrm{OvI}), 76 \%$ of the LOS have ratios smaller than -1 , while the remaining LOS (24\%) have $-1<$ $N(N \mathrm{v}) / N(O \mathrm{vI}) \leqslant-0.5$ (red histogram).

Using the above ions ratios, we further explore the ionization mechanisms responsible for their formation. Hence, in Figure 5 we compare the variation of $\log [N(\mathrm{C}$ IV $) / N(\mathrm{O}$ VI $)]$ versus $\log [N(\mathrm{~N} v) / N(\mathrm{O}$ vI $)]$ inside the $\mathrm{LB}$ at present time with the expected ratios from conductive interfaces (CIs; Borkowski et al. 1990), radiative cooling flows (RC; Edgar \& Chevalier 1986), turbulent mixing layers (TMLs; Slavin et al. 1993), and shock ionization (SI; Dopita \& Sutherland 1996). The model predictions have been corrected for the solar abundances used in the present paper (AG89) following Fox et al. (2004). The figure shows that (i) most of the data points are concentrated in the region $N(C \mathrm{IV}) / N(O \mathrm{VI}) \in[-2.4,-1.5]$ and $N(N \mathrm{v}) / N(O \mathrm{vI}) \in[-1.7,-1]$ and around the collisional ionization predictions (solid black line labeled CIE), and (ii) the dispersion of data points increases with increasing LOS length, and fall within the CIs and TMLs predictions models. As the simulation does not include heat conduction, the dispersion of the data points within the CI region results from the entrainment of the gas elements with different temperatures, which, by sliding past each other, generate shear leading to the generation of Kelvin-Helmholtz instabilities that mix the two gases in an intermediate-temperature mixing layer, which is still hot enough to generate ions such $\mathrm{C}$ IV and $\mathrm{N} v$ by electron collisions (Begelman \& Fabian 1990). That is, TMLs are playing the role of CIs.

We recall that the $\mathrm{C}, \mathrm{N}$, and $\mathrm{O}$ abundances in AG89 have been significantly updated by Asplund et al. (2005), from $\log A_{C}^{\odot}=-3.44, \log A_{N}^{\odot}=-3.95$, and $\log A_{O}^{\odot}=-3.07$, to $-3.61,-4.22$, and -3.34 , respectively. Following Fox et al. (2004), the correction of the above results to the new abundances implies an increase in $\log [N(\mathrm{C}$ IV $) / N(\mathrm{O}$ vi $)]$ of 0.1 , no changes occur on $\log [N(\mathrm{~N} v) / N(\mathrm{O} \mathrm{VI})]$. This translates into a shift of 0.1 

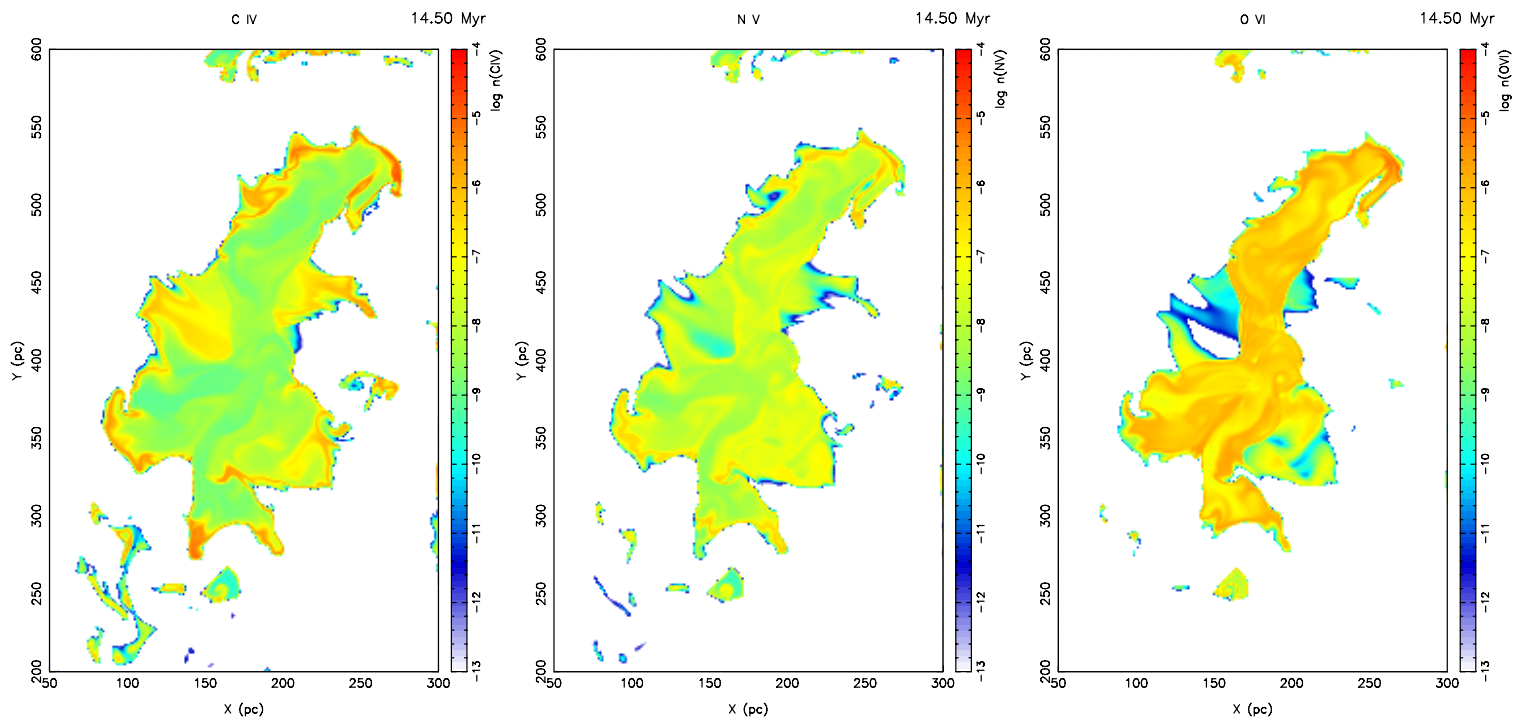

Figure 2. C Iv, N v, and O vi column distributions inside the LB cavity at 14.5 Myr.
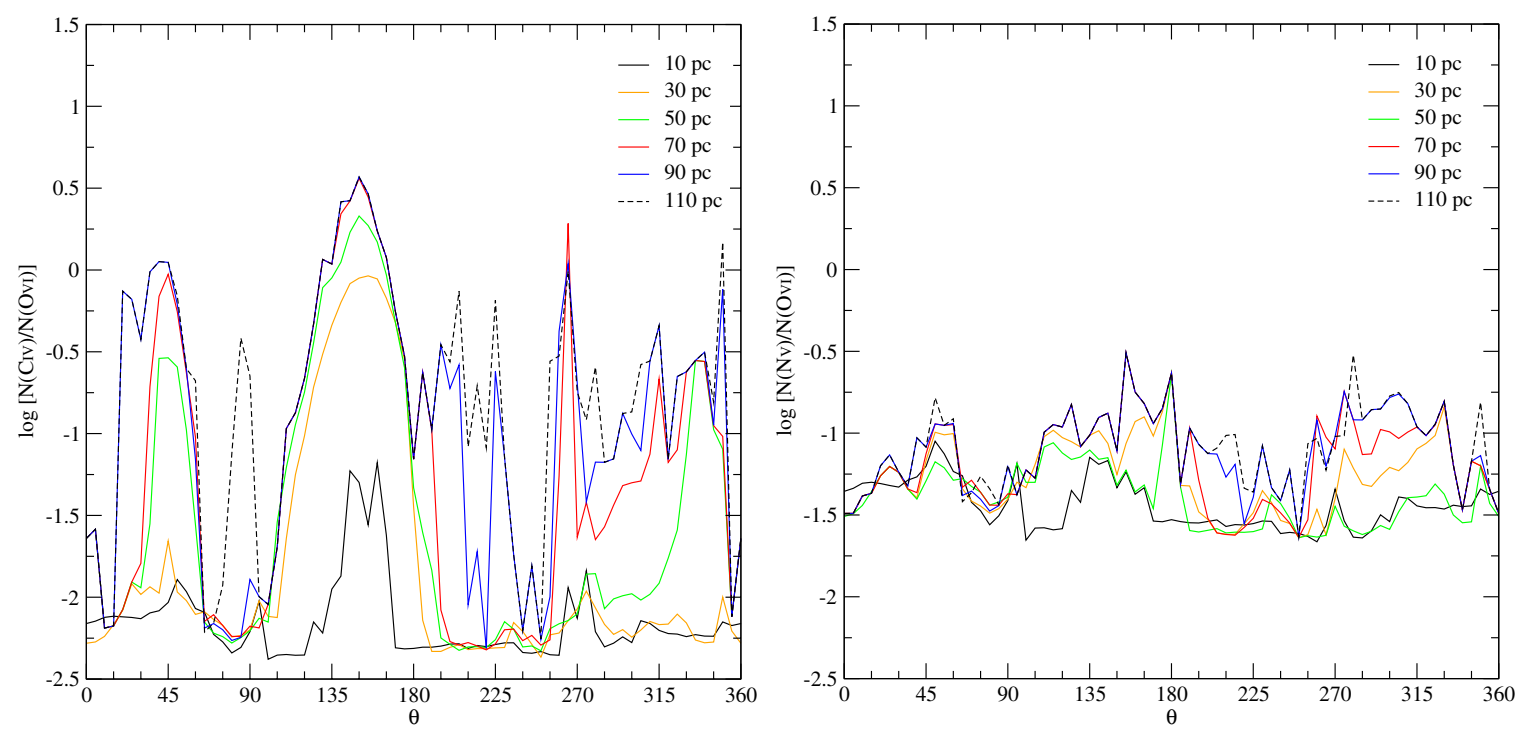

Figure 3. Column density ratios $N(\mathrm{C} \mathrm{IV}) / N(\mathrm{O} \mathrm{vI})$ (left panel) and $N(\mathrm{~N} \mathrm{v}) / N(\mathrm{O}$ vI) (right panel) measured inside the simulated LB as a function of angle for different distances from the vantage point (for LOS distribution see Figure 1).

along the $y$-axis in Figure 5, with no changes on our conclusions. In a forthcoming paper, we present the evolution of the LB using most recent abundance tables of Asplund et al. (2005).

\section{DISCUSSION AND FINAL REMARKS}

In this Letter, we discuss the distribution of the $\mathrm{C}$ IV and $\mathrm{N} \mathrm{V}$ ions inside the simulated LB at the present time; that is, about $0.5 \mathrm{Myr}$ after the last SN occurrence in the cavity. Our results are consistent with the $3 \sigma$ upper limits for $N(\mathrm{C}$ IV $)$ and $N(\mathrm{~N} \mathrm{v})$ derived from CoPERNICUs (York 1977), IUE (Bruhweiler et al. 1980), and GHRS-HST (Bertin et al. 1995; Huang et al. 1995) observations toward stars located up to $150 \mathrm{pc}$ from the Sun. These observations point to a lack of $\mathrm{C}$ IV and $\mathrm{N} v$ ions in the $\mathrm{LB}$ translating into $\log [N(\mathrm{C}$ IV $) / N(\mathrm{O}$ VI $)]$ and $\log [N(\mathrm{~N} \mathrm{v}) / N(\mathrm{O} \mathrm{vI})]$ $<-1.0$.

The increase in the column density ratios with distance inside the LB is indicative of the importance of turbulent mixing. In fact, turbulent motions are larger near the shell where mass flow into the cavity occurs. Hence, shear is further enhanced and turbulent diffusion progresses inward. Weak shocks, resulting from the successive explosions, cross the cavity within a sound crossing time, strongly compressing and heating the surrounding shell, thereby promoting further mass inflow. We also note that due to the interaction between the LB and Loop I, the development of Rayleigh-Taylor instabilities will cause the formation of local clouds and also enhance mass loading (compared to Breitschwerdt et al. 2000). All this leads to the redistribution of the energy inside the cavity and to turbulent mixing, thereby reducing the temperature between hotter and cooler regions (Begelman \& Fabian 1990), resulting in intermediate temperatures that are still large enough to generate ions such as $\mathrm{C} I V, \mathrm{Nv}$, and $\mathrm{O}$ VI. As heat conduction depends on strong temperature gradients, the timescale for it to become dominant is in general larger than that of turbulent mixing, which occurs on all scales and is driven by the nonlinear term in the Navier-Stokes equation. Note that the turbulent viscosity is considerably enhanced by a factor of the order of 


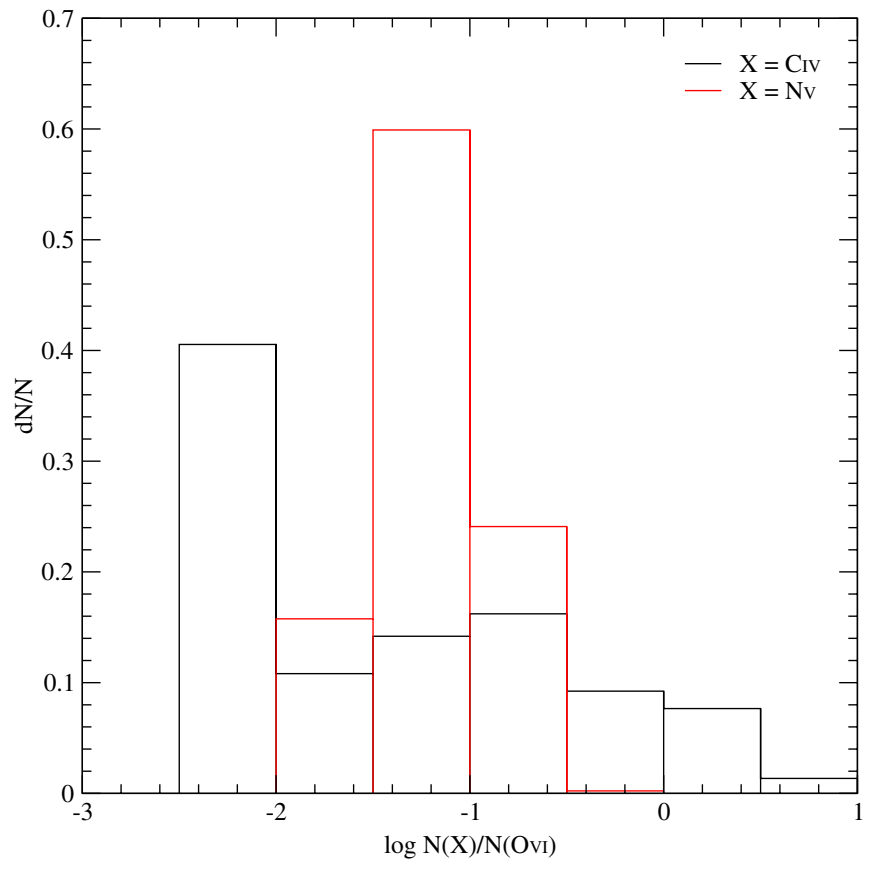

Figure 4. Histogram of the LOS (with length $120 \mathrm{pc}$ ) measurements of the column density ratios $N(\mathrm{C} \mathrm{IV}) / N(\mathrm{O}$ vI) (black) and $N(\mathrm{~N} \mathrm{v}) / N(\mathrm{O}$ vI) (red).

the Reynolds number, which is about $10^{5}-10^{7}$ (Elmegreen \& Scalo 2004) in the ISM. Thus, the role of heat conduction is negligible in the redistribution of the energy, and therefore does not affect the column density of the observed ions.

Recently, it has been shown that charge exchange reactions between solar wind ions and local neutral plasma (most likely inside the heliosphere) can account for some fraction of the observed SXR background (Cravens 2000; Lallement 2004). However, this contribution is decreasing with increasing galactic latitude. In their most recent paper, Koutroumpa et al. (2008) show that it may be possible to attribute all of the $3 / 4 \mathrm{keV}$ emission to SWCX. However, there still remains a noticeable deficit of $1 / 4 \mathrm{keV}$ band emission that cannot be explained by SWCX. It is an advantage of our model-in contrast to the displacement model or others - that it can easily accommodate lower SXR contributions, simply by constraining further the age of the LB by the time elapsed since the last $\mathrm{SN}$ explosion.

\section{REFERENCES}

Anders, E., \& Grevesse, N. 1989, Geochim. Cosmochim. Acta, 53, 197 (AG89) Asplund, M., Grevesse, N., \& Sauval, A. J. 2005, in ASP Conf. Ser. 336, Cosmic Abundances as Records of Stellar Evolution and Nucleosynthesis in honor of David L. Lambert, ed. T. G. Barnes III \& F. N. Bash (San Francisco, CA: ASP), 25

Begelman, M. C., \& Fabian, A. C. 1990, MNRAS, 244, 26

Berghöfer, T., \& Breitschwerdt, D. 2002, A\&A, 390, 299

Bertin, P., Vidal-Madjar, A., Lallement, R., Ferlet, R., \& Lemoine, M. 1995, A\&A, 302, 889

Borkowski, K. L., Balbus, S. A., \& Fristrom, C. C. 1990, ApJ, 355, 501

Breitschwerdt, D., \& de Avillez, M. A. 2006, A\&A, 452, L1 (BA06)

Breitschwerdt, D., Egger, R., \& Freyberg, M. J. 2000, A\&A, 361, 303

Breitschwerdt, D., \& Schmutzler, T. 1994, Nature, 371, 774

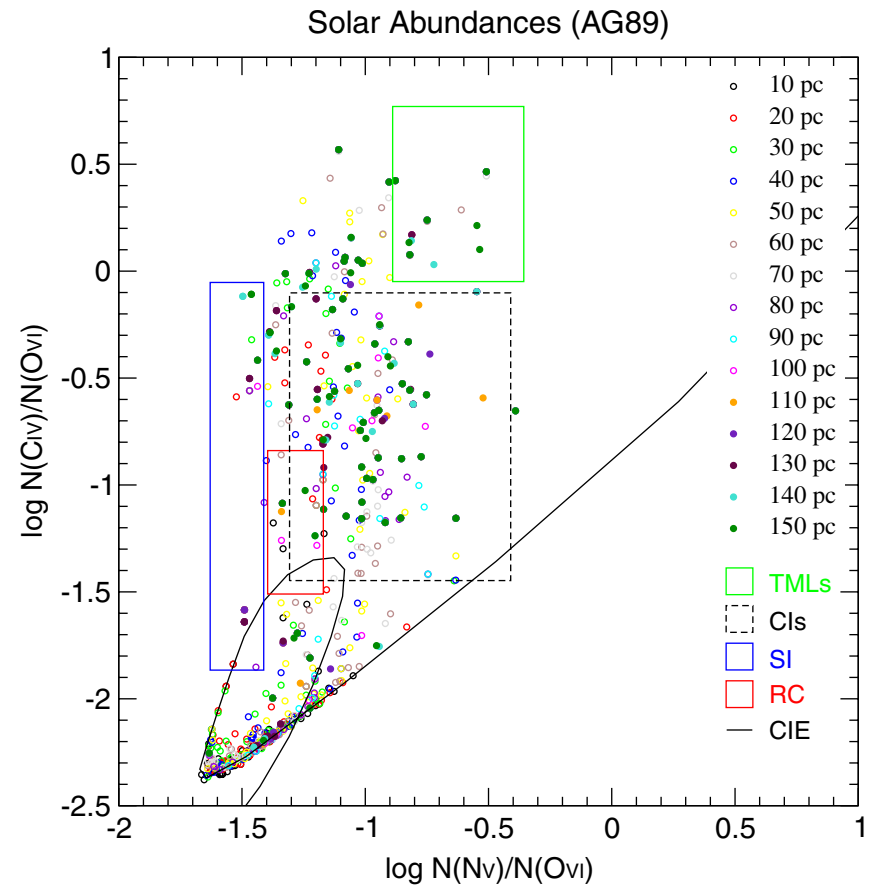

Figure 5. Comparison of $\log [N(\mathrm{C}$ Iv $) / N(\mathrm{O}$ vI $)]$ vs. $\log [N(\mathrm{~N} v) / N(\mathrm{O} v \mathrm{v})]$ measured column density ratios, CIE predictions, and the theoretical estimates from different ionization mechanisms models: TMLs (Slavin et al. 1993), SI (Dopita \& Sutherland 1996), CIs (Borkowski et al. 1990), and RC (Edgar \& Chevalier 1986). Due to the changes in abundances since the first publication of CI, RC, and TMLs, their predictions have been corrected for the solar abundances used in this Letter.

Bruhweiler, F. C., Kondo, Y., \& McCluskey, G. E. 1980, ApJ, 237, 19

Cravens, T. E. 2000, ApJ, 532, L153

Dalgarno, A., \& Mc Cray, R. A. 1972, ARA\&A, 10, 375

de Avillez, M., \& Breitschwerdt, D. 2005, ApJ, 634, L65

Dopita, M. A., \& Sutherland, R. S. 1996, ApJS, 102, 161

Drake, J. J., \& Testa, P. 2005, Nature, 436, 525

Edgar, R. J., \& Chevalier, R. A. 1986, ApJ, 310, L27

Egger, R. 1998, in IAU Colloq. 166, Lecture Notes in Physics 506, The Local Bubble and Beyond, ed. D. Breitschwerdt, M. J. Freyberg, \& J. Truemper (Berlin: Springer), 287

Egger, R., \& Aschenbach, B. 1995, A\&A, 294, L25

Elmegreen, B. G., \& Scalo, J. 2004, ARA\&A, 42, 211

Fox, A. J., Savage, B. D., Wakker, B. P., Richter, P., Sembach, K. R., \& Tripp, T. M. 2004, ApJ, 602, 738

Fuchs, B., Breitschwerdt, D., de Avillez, M. A., Dettbarn, C., \& Flynn, C. 2006, MNRAS, 373, 993

Gnat, O., \& Sternberg, A. 2007, ApJS, 168, 213

Huang, J.-S., Songaila, A., Cowie, L. L., \& Jenkins, E. B. 1995, ApJ, 450, 163

Hurwitz, M., Sasseen, T. P., \& Sirk, M. M. 2005, ApJ, 623, 911

Jenkins, E. B. 1978, ApJ, 219, 845

Koutroumpa, D., Lallement, R., Kharchenko, V., \& Dalgarno, A. 2008, SSRv, 143,217

Lallement, R. 2004, A\&A, 422, 391

McCammon, D., et al. 2002, ApJ, 576, 188

Oegerle, W. R., Jenkins, E. B., Shelton, R. L., Bowen, D. V., \& Chayer, P. 2005, ApJ, 622, 377

Savage, B. D., \& Lehner, N. 2006, ApJS, 162, 134

Shelton, R. 2003, ApJ, 589, 261

Shelton, R., \& Cox, D. P. 1994, ApJ, 434, 599

Slavin, J. D., Shull, J. M., \& Begelman, M. C. 1993, ApJ, 407, 83

Stothers, R. 1972, ApJ, 175, 431

York, D. G. 1977, ApJ, 213, 43 